\title{
Barycentric decomposition of quantum measurements in finite dimensions
}

\author{
G. Chiribella, G. M. D'Ariano, and D.-M. Schlingemann \\ Quantum Information Theory Group, Dipartimento di Fisica A. Volta, Università di Pavia, via Bassi 6, 27100 Pavia, \\ Italy \\ INFN, Sezione di Pavia, via Bassi 6, 27100 Pavia, Italy. \\ ISI Foundation, Quantum Information Theory Unit, Viale S. Severo 65, 10133 Torino, Italy and \\ Institut für Mathematische Physik, Technische Universität Braunschweig, Mendelssohnstraße 3, 38106 Braunschweig, \\ Germany
}

\begin{abstract}
We analyze the convex structure of the set of positive operator valued measures (POVMs) representing quantum measurements on a given finite dimensional quantum system, with outcomes in a given locally compact Hausdorff space. The extreme points of the convex set are operator valued measures concentrated on a finite set of $k \leq d^{2}$ points of the outcome space, $d<\infty$ being the dimension of the Hilbert space. We prove that for second countable outcome spaces any POVM admits a Choquet representation as the barycenter of the set of extreme points with respect to a suitable probability measure. In the general case, Krein-Milman theorem is invoked to represent POVMs as barycenters of a certain set of POVMs concentrated on $k \leq d^{2}$ points of the outcome space.
\end{abstract}

\section{INTRODUCTION}

In the modern formalism of Quantum Mechanics the statistical description of a measurement is provided by the concept of positive operator valued measure (POVM) $(\underline{6} ; 13 ;$; 16; 17), whose introduction in the literature on quantum probability dates back to the seminal papers by Davies and Lewis (14) and Holevo (18). A POVM associates to any possible event in a quantum experiment a positive semidefinite operator on the Hilbert space of the measured system, in such a way that the probability of the event is given by the expectation value of the corresponding operator on the quantum state describing the system preparation. The concept of POVM generalizes, as far as it concerns the statistical aspects, the traditional concept of "observable" by von Neumann (37), which turned out to be a too restrictive idealization to efficiently describe actual experimental settings (such as the heterodyne measurement in quantum optics (24)), and even to give a realistic modeling of photon-counting in the presence of losses (28).

In the case of finite dimensional quantum systems, the number of different outcomes of a von Neumann observable must be finite, as the number of eigenvalues of a self-adjoint operator cannot exceed the dimension $d<\infty$ of the Hilbert space. Based on this observation, it is commonly argued that all quantities measured on finite dimensional systems must be intrinsically discrete or "quantized". For example, when measured, a spin $j$ particle would be found in only $d_{j}=2 j+1$ possible spatial configurations, corresponding to the possible values of the angular momentum along a given quantization axis. The limitation on the number of possible values, however, only holds for von Neumann measurements, which are a very particular subset of all possible measurements in the statistical model of Quantum Mechanics (19). If one considers arbitrary POVMs, then there is no bound on the number of outcomes in an experiment, a number which can be even uncountably infinite, despite the Hilbert space dimension is finite. This is indeed the case for the optimal measurement of the spatial orientation of a spin $j$ that has been devised in Ref. (20): in this measurement any direction in the unit sphere is a possible outcome of the experiment.

From an operational point of view, a statement about the discreteness of physical quantities of finite dimensional quantum systems cannot rely on the concept of von Neumann observables. The question is then: Is it possible to give a rigorous account to the intuitive idea that the information carried by finite dimensional systems is intrinsically discrete? This intuitive idea is indeed supported by several features, such as the existence of fundamental dimension-dependent limits to the precision of phase measurements on atomic clocks (7), to the extraction of directional information from quantum gyroscopes (10), and to the maximum accessible information in a coding-decoding scheme (21). Since all mentioned limits arise in optimization problems where the goal is to find quantum measurements that maximize some convex figure of merit, it is natural to analyze the convex structure of the set of measurements (POVMs) with given outcome space, expecting that the discrete nature of information in finite dimensional systems will be unveiled by the characterization of extreme points.

This paper fully characterizes the convex structure of the set of POVMs with outcomes in a given locally compact Hausdorff space $Y$, by $i$ ) identifying the extreme points, and ii) proving a representation of arbitrary POVMs as barycenters of sets of POVMs with finite outcomes. We will first show that any extreme positive operator valued measure is concentrated on a finite number $k$ of points, with $k$ not greater than $d^{2}$, the square of the Hilbert space dimension. If $\Delta \subseteq Y$ is a possible event and $M(\Delta)$ is the corresponding POVM operator, this means that an extreme 
POVM $M$ must be of the form

$$
M(\Delta)=\sum_{i=1}^{k} \chi_{\Delta}\left(y_{i}\right) P_{i},
$$

where $\chi_{\Delta}$ is the indicator function of the set $\Delta,\left\{y_{i} \in Y \mid i=1,2, \ldots, k\right\}$ is a finite set of distinct points, and $\left\{P_{i} \mid i=1,2, \ldots, k\right\}$ is a finite set of operators forming an extreme POVM with finite outcome space $X=\{1,2, \ldots, k\}$, i.e. $P_{i} \geq 0, \sum_{i} P_{i}=\mathbb{1}_{d}$. Operationally, this means that any extreme POVM $P$ can be realized by first performing a quantum measurement with finite set of outcomes $X=\{1,2, \ldots, k\}$, and then by injecting the result $i \in X$ in the outcome space $Y$ via a post-processing rule $i \rightarrow y_{i}$. This result reduces the characterization of the extreme POVMs with locally compact outcome space to the simpler characterization of extreme POVMs with finite outcomes, which is has been extensively studied in the works by Störmer (32), Parthasarathy (30), and D'Ariano, Lo Presti, and Perinotti (12). Finally, we exploit Choquet theorem to show that for second countable outcome spaces any POVM can be represented as a barycenter of the set of extreme POVMs. For general outcome spaces a barycentric representation in terms of the closure of the set of extreme points is obtained instead by means of Krein-Milman theorem. In both cases, combining the barycentric decomposition with the characterization of the extreme POVMs shows that for finite dimensional quantum systems any measurement with a continuum of outcomes is nothing but the randomized choice, according to a continuous probability distribution, of a certain set of measurements with finite outcomes. In this sense, the continuum of outcomes is simply equivalent to the presence of classical randomness controlling the choice of the measuring apparatus. This provides the rigorous and complete proof of the results presented in Ref. (11).

It is worth stressing that all our results are derived for finite dimensional Hilbert spaces, while in infinite dimensions the situation is dramatically different. Indeed it is well known that von Neumann observables always correspond to extreme POVMs, and any observable with continuous spectrum is an example of extreme POVM with genuinely uncountable outcome space, despite the Hilbert space has a countable orthonormal basis. Moreover, a remarkable feature in infinite dimensions is that von Neumann observables are dense in the set of POVMs with given outcome space (22).

The paper is organized as follows: In Section $\amalg$ we provide the basic notation and definitions. In particular, we highlight the equivalence between POVMs and regular operator valued expectations (OVEs), a class of positive maps that will be extensively used in the statement and in the derivation of the main results. Regular operator valued expectations coincide with what is known as quantization maps in the literature on geometric quantization (1; 25), namely positive maps from functions on a classical phase-space to operators on the system's Hilbert space. It is worth stressing that the present paper can be read as well as a characterization of the extreme quantization maps for finite dimensional quantum systems, along with a barycentric representation of arbitrary quantization maps. The characterization of extreme POVMs/regular OVEs is carried out in Sec. III Section IV presents a few topological properties that will be useful for deriving barycentric decompositions. Finally, Section $\nabla$ is devoted to the proof of barycentric representations of POVMs and regular OVEs, first in the case of second countable outcome spaces, and then in the general case.

\section{POSITIVE OPERATOR VALUED MEASURES AND EXPECTATIONS}

\section{A. Positive operator valued measures}

In the following $\mathrm{M}_{d}$ and $\mathrm{M}_{d}^{*}$ will denote the $\mathrm{C}^{*}$-algebra of $d \times d$ complex matrices and the Banach space of linear functionals on $\mathrm{M}_{d}$, respectively.

Definition 1 Let $Y$ be a measure space with $\sigma$-algebra $\sigma(Y)$. A positive operator valued measure (POVM) in dimension $d<\infty$ is a map $M: \sigma(Y) \rightarrow \mathrm{M}_{d}$ that assigns to each measurable set $\Delta \in \sigma(Y)$ an operator $M(\Delta) \in \mathrm{M}_{d}$ satisfying the following conditions:

Positivity: $M(\Delta) \geq 0 \quad \forall \Delta \in \sigma(Y)$

Normalization: $M(Y)=\mathbb{1}_{d}$, with $\mathbb{1}_{d} \in \mathrm{M}_{d}$ the identity matrix.

$\sigma$-Additivity: $M\left(\cup_{i \in \mathbb{N}} \Delta_{i}\right)=\sum_{i \in \mathbb{N}} M\left(\Delta_{i}\right)$ for any countable family of mutually disjoint sets $\left\{\Delta_{i} \in \sigma(Y) \mid i \in \mathbb{N}\right\}$, where the series converges weakly.

Throughout this paper the measure space $Y$ will be always a locally compact Hausdorff space, and $\sigma(Y)$ will always denote the Borel $\sigma$-algebra. The term POVM will be used as a synonymous of regular Borel POVM, as defined in the following: 
Definition 2 Let $Y$ be a locally compact Hausdorff space with Borel $\sigma$-algebra $\sigma(Y)$. A Borel POVM $M$ is called regular if the condition

$$
M(\Delta)=\sup \{M(K) \mid K \subseteq \Delta, K \text { compact }\}
$$

is fulfilled for any Borel set $\Delta \in \sigma(Y)$.

The set of regular Borel POVMs is a convex set, denoted by $\mathscr{M}(Y, d)$, and will be the focus of our investigation.

In quantum mechanics, any POVM yields the probabilities of events occurring in a particular experimental setup. The elements of the space $Y$ are the possible outcomes of the experiment, and $Y$ is accordingly referred to as outcome space. The possible events are measurable subsets of $Y$, the subset $\Delta$ corresponding to the event "the outcome of the experiment belongs to $\Delta$ ". The states of a quantum system with finite dimensional Hilbert space $\mathcal{H} \simeq \mathbb{C}^{d}$ are positive normalized functionals over the $\mathrm{C}^{*}$-algebra of complex matrices $\mathrm{M}_{d}$. For a quantum system prepared in the state $\rho \in \mathrm{M}_{d}^{*}$ the probability of the event $\Delta$ is given by the Born rule

$$
p(\Delta)=\rho(M(\Delta)) .
$$

Accordingly, the POVM $M$ assigns to every quantum state $\rho$ a classical probability distribution $m_{\rho}$ via the relation $m_{\rho}(\Delta)=\rho(M(\Delta))$. Any bounded measurable function $f$ can by averaged with respect to $m_{\rho}$, thus yielding the expectation value

$$
\mathbb{E}_{m_{\rho}}(f)=\int_{Y} m_{\rho}(\mathrm{d} y) f(y)=\int_{Y} \rho(M(\mathrm{~d} y)) f(y) .
$$

The expectation $\mathbb{E}_{m_{\rho}}(f)$ in Eq. (4) can be extended by linearity to a unique functional on $\mathrm{M}_{d}^{*}$, i.e. to a unique operator $E(f) \in \mathrm{M}_{d}$ satisfying the relation:

$$
\rho(E(f))=\mathbb{E}_{m_{\rho}}(f) \quad \forall \rho \in \mathrm{M}_{d}^{*} .
$$

The map $E: f \mapsto E(f)$ can be viewed as an operator valued expectation: indeed, comparing Eqs. (4) and (5) we obtain

$$
E(f)=\int M(\mathrm{~d} y) f(y)
$$

the integral converging in the weak operator topology (4).

\section{B. Operator valued expectations}

Dealing with locally compact Hausdorff spaces, it is convenient to focus our attention to the $\mathrm{C}^{*}$-algebra $\mathscr{C}_{0}(Y)$ of continuous functions vanishing at infinity, equipped with the sup-norm $\|f\|=\sup _{y \in Y}|f(y)|$. In the following, we will consider $\mathscr{C}_{0}(Y)$ as a subalgebra of the unital $\mathrm{C}^{*}$-algebra of functions that are constant at infinity

$$
\begin{aligned}
\overline{\mathscr{C}_{0}}(Y) & =\mathscr{C}_{0}(Y) \oplus \mathbb{C} \\
& =\left\{a f+b \mathbb{1}_{Y} \mid f \in \mathscr{C}_{0}(Y), a, b \in \mathbb{C}\right\},
\end{aligned}
$$

where $\mathbb{1}_{Y}$ is the constant function $\mathbb{1}_{Y}(y)=1 \forall y \in Y$. Moreover, we will extensively use that fact that the $\mathrm{C}^{*}$-algebra $\overline{\mathscr{C}}_{0}(Y)$, obtained by adding the unit to $\mathscr{C}_{0}(Y)$, is naturally isomorphic to $\mathscr{C}(\bar{Y})$, the $\mathrm{C}^{*}$-algebra of continuous functions on the one-point compactification $\bar{Y}=Y \cup\{\infty\}$ (38).

Definition 3 An operator valued expectation (OVE) in dimension $d<\infty$ is a map $E: \overline{\mathscr{C}}_{0}(Y) \rightarrow \mathrm{M}_{d}$ that assigns to any function $f \in \overline{\mathscr{C}}_{0}(Y)$ an operator $E(f) \in \mathrm{M}_{d}$ satisfying the following conditions:

Positivity: $E(f) \geq 0 \quad \forall f \geq 0$

Normalization: $E\left(\mathbb{1}_{Y}\right)=\mathbb{1}_{d}$.

Operator valued expectations form a convex subset of the set $\mathscr{B}(Y, d)$ of bounded maps from $\overline{\mathscr{C}}_{0}(Y)$ to $\mathrm{M}_{d}$, where the norm is defined by

$$
\|E\|=\sup _{f \in \overline{\mathscr{C}}_{0}(Y):\|f\|=1}\|E(f)\|,
$$

$\|O\|$ denoting the operator norm of $O \in \mathrm{M}_{d}$. The set of all operator valued expectations will be denoted by $\mathscr{E}(Y, d)$. 
Remark 1 Since the domain of the positive map $E \in \mathscr{E}(Y, d)$ is the abelian algebra $\overline{\mathscr{C}_{0}}(Y), E$ is automatically completely positive (27). Therefore, for any OVE $E \in \mathscr{E}(Y, d)$ we have

$$
\|E\|=\sup _{0 \leq f \leq \mathbb{1}_{Y}}\|E(f)\|=\left\|E\left(\mathbb{1}_{Y}\right)\right\|=\left\|\mathbb{1}_{d}\right\|=1 .
$$

This shows that the set $\mathscr{E}(Y, d)$ is contained in the intersection between the cone of positive maps and the unit ball in $\mathscr{B}(Y, d)$. Notice that such an intersection also contains positive maps that are not OVEs: not any positive map $E$ with $\|E\|=1$ satisfies $E\left(\mathbb{1}_{Y}\right)=\mathbb{1}_{d}$.

Remark 2 Since the unital algebra $\overline{\mathscr{C}}_{0}(Y)$ can be identified with $\mathscr{C}(\bar{Y})$, the set of OVEs $\mathscr{E}(Y, d)$ can be identified with the set of OVEs $\mathscr{E}(\bar{Y}, d)$, namely $\mathscr{E}(Y, d) \simeq \mathscr{E}(\bar{Y}, d)$. In the following we will make often exploit this identification.

\section{Relation between POVMs and OVEs}

Each POVM $M \in \mathscr{M}(Y, d)$ induces an OVE $E \in \mathscr{E}(Y, d)$ via the relation (6). The converse, however, is not straightforward, as in the definition of OVE there are no requirements entailing $\sigma$-additivity and regularity of measures. This motivates the following definition:

Definition 4 An $O V E E \in \mathscr{E}(Y, d)$ is called regular if

$$
\sup \left\{E(f) \mid f \in \mathscr{C}_{0}(Y), 0 \leq f \leq 1\right\}=\mathbb{1}_{d}
$$

The subset of regular OVEs will be denoted by $\mathscr{R}(Y, d)$. Notice that for compact outcome spaces $Y$ all OVEs are regular, namely $\mathscr{R}(Y, d) \equiv \mathscr{E}(Y, d)$.

As already mentioned in the introduction, regular OVEs are also known as quantization maps in the literature on geometric quantization (1; 25). The relation between between regular OVEs (quantization maps) and POVMs is a well known fact in such a literature (see e.g. (25)), and is reported here for completeness of presentation.

Theorem 1 (Characterization of regular OVEs) Let $Y$ be a locally compact Hausdorff space. An OVE E $\in$ $\mathscr{E}(Y, d)$ is regular if and only if there exists a POVM $M_{E} \in \mathscr{M}(Y, d)$ such that

$$
E(f)=\int M_{E}(\mathrm{~d} y) f(y) .
$$

The above equation sets a one-to-one affine correspondence between $\mathscr{R}(Y, d)$ and $\mathscr{M}(Y, d)$.

Proof. Let $E$ be an OVE. Then for any state $\rho \in \mathrm{M}_{d}^{*}$ the composition $\rho \circ E$ defines a state on $\overline{\mathscr{C}}_{0}(Y)$. Moreover, $E$ is regular if and only if the restriction of $\rho \circ E$ to the ideal $\mathscr{C}_{0}(Y)$ satisfies $\left\|\left.\rho \circ E\right|_{\mathscr{C}_{0}(Y)}\right\|=1$, namely if and only if $\left.\rho \circ E\right|_{\mathscr{C}_{0}(Y)}$ is a state on $\mathscr{C}_{0}(Y)$. By Riesz-Markov theorem $(9 ;$;3) $)$, states on $\mathscr{C}_{0}(Y)$ are uniquely represented by regular probability measures on $Y$. Therefore $E$ is regular if and only if for any state $\rho$ there exists a unique probability measure $m_{E, \rho}$ such that $\rho(E(f))=\int m_{E, \rho}(\mathrm{d} y) f(y), \forall f \in \overline{C_{0}}(Y)$. Since the map $\rho \rightarrow m_{E, \rho}(\Delta)$ is convex linear in $\rho$, it extends uniquely to a linear functional on $\mathrm{M}_{d}^{*}$, i.e. to an operator $M_{E}(\Delta) \in \mathrm{M}_{d}$. The map $\Delta \rightarrow M_{E}(\Delta)$, uniquely determined by this construction, is clearly a POVM. Hence, $E$ is regular if and only if there exists a POVM $M_{E}$ such that $E(f)=\int_{Y} M_{E}(\mathrm{~d} y) f(y)$. Of course, $M_{E}=M_{F}$ implies $E=F$.

Theorem 1 also provides a characterization of the whole set $\mathscr{E}(Y, d)$ :

Corollary 1 Let $Y$ be a locally compact Hausdorff space, and let $\bar{Y}$ be its one-point compactification. Then the following chain of isomorphisms holds

$$
\mathscr{E}(Y, d) \simeq \mathscr{E}(\bar{Y}, d) \simeq \mathscr{M}(\bar{Y}, d)
$$

Proof. Since $\overline{\mathscr{C}_{0}}(Y)$ is isomorphic to $\mathscr{C}(\bar{Y})$, one has the natural isomorphism $\mathscr{E}(Y, d) \simeq \mathscr{E}(\bar{Y}, d)$. Moreover, since $\bar{Y}$ is compact, one has $\mathscr{E}(\bar{Y}, d) \equiv \mathscr{R}(\bar{Y}, d)$, and, due to Theorem $\mathbb{1}, \mathscr{R}(\bar{Y}, d) \simeq \mathscr{M}(\bar{Y}, d)$. 


\section{Convexity and topology}

The sets $\mathscr{E}(Y, d)$ and $\mathscr{R}(Y, d) \simeq \mathscr{M}(Y, d)$ possess a natural convex structure, namely the convex combination of two (regular) OVEs is a (regular) OVE. Operationally, the convex combination of two quantum measurements corresponds to a random choice of the corresponding measurement apparatuses with suitable probabilities. The extreme OVEs are those which cannot be decomposed into nontrivial convex combinations:

Definition 5 An OVE $E \in \mathscr{E}(Y, d)$ is extreme if for any couple of OVEs $E_{+}, E_{-} \in \mathscr{E}(Y, d)$ the equality E $=$ $1 / 2\left(E_{+}+E_{-}\right)$implies $E_{+}=E_{-}=E$.

Similarly one can define the extreme regular OVEs. The extreme points of $\mathscr{E}(Y, d)$ and $\mathscr{R}(Y, d)$ will be denoted by $\partial \mathscr{E}(Y, d)$ and $\partial \mathscr{R}(Y, d)$, respectively.

The notion of finite convex combination can be generalized to the notion of barycenter, that includes the possibility of infinite combinations with arbitrary probability distributions. For this generalization, however, one has to first specify a topology on the set of OVEs. We will consider here the weak*-topology induced by the family of seminorms

$$
w_{\rho, f}(E)=|\rho(E(f))|
$$

with $\rho \in \mathrm{M}_{d}^{*}$ and $f \in \overline{\mathscr{C}_{0}}(Y)$. This topology has a direct operational interpretation in quantum mechanics: what can be tested in experiments are indeed the expectation values $\rho(E(f))$ where $\rho$ is the state of the quantum system, $E$ describes the measurement, and $f$ is a function of the outcome. If the expectation values $\rho\left(E_{n}(f)\right)$ obtained in a sequence of measurements $\left\{E_{n}\right\}$ converge to $\rho(E(f))$ for any state $\rho$ and any function $f$, then the sequence of measurements $\left\{E_{n}\right\}$ converges to $E$. Accordingly, the weak ${ }^{*}$-closure $\overline{\mathscr{U}}$ of a set of quantum measurements contains all OVEs that can be arbitrarily approximated with measurements in $\mathscr{U}$ in the sense of expectation values.

Let $\sigma(\mathscr{E}(Y, d))$ be the Borel $\sigma$-algebra generated by weak*-open sets.Then we have the following definition:

Definition 6 Let $p$ be a probability distribution on $\sigma(\mathscr{E}(Y, d))$ and $\mathscr{U} \in \sigma(\mathscr{E}(Y, d))$ be a Borel set. An OVE $E$ is the barycenter of $\mathscr{U}$ with respect to $p$, denoted by

$$
E=\int_{\mathscr{U}} p(\mathrm{~d} F) F
$$

if for any $\rho \in \mathrm{M}_{d}^{*}$ and for any $f \in \overline{\mathscr{C}}_{0}(Y)$ the following relation holds:

$$
\rho(E(f))=\int_{\mathscr{U}} p(\mathrm{~d} F) \rho(F(f)) .
$$

Notice that the integral in Eq. (15) is well defined since the expectation value $\rho(F(f))$ is by definition a weakly*continuous function of $F$, and therefore can be integrated with respect to any Borel measure $p(\mathrm{~d} F)$.

\section{CHARACTERIZATION OF EXTREME POVMS}

\section{A. Existence of densities for OVEs in finite dimensions}

We first prove that every regular OVE admits a density with respect to a finite measure on $Y$.

Lemma 1 For any regular $O V E E \in \mathscr{R}(Y, d)$ there exist a regular finite measure $\mu_{E}$ on $Y$ and a positive density function $D_{E} \in L_{\infty}\left(Y, \mu_{E}\right) \otimes \mathrm{M}_{d}$ such that for any $f \in \overline{\mathscr{C}_{0}}(Y)$

$$
E(f)=\int \mu_{E}(\mathrm{~d} y) D_{E}(y) f(y) .
$$

The density function $D_{E}$ has unit trace, namely $\operatorname{tr}\left[D_{E}(y)\right]=1 \mu_{E}$-almost everywhere.

Proof. Let $\operatorname{tr}$ be the trace on $\mathrm{M}_{d}$. Then $\hat{\mu}_{E}:=\operatorname{tr} \circ E$ is a positive functional with norm $\left\|\hat{\mu}_{E}\right\|=d$. Since $E$ is regular, by Riesz-Markov theorem $\hat{\mu}_{E}$ can be represented by a regular finite measure $\mu_{E}$ on $Y$. Moreover, the dominance relation $E \leq \hat{\mu}_{E} \mathbb{1}_{d}$ holds. Indeed, for any positive function $f$ one has $E(f) \leq\|E(f)\| \mathbb{1}_{d} \leq \operatorname{tr}[E(f)] \mathbb{1}_{d}=$ $\hat{\mu}_{E}(f) \mathbb{1}_{d}$. The Radon-Nikodym Theorem for OVEs [Lemma 11 of the Appendix] then guarantees the existence 
of a positive density $D_{E} \in L_{\infty}\left(Y, \mu_{E}\right) \otimes \mathrm{M}_{d}$, namely an operator valued function $D_{E}(y)$ satisfying the relation $E(f)=\int \mu_{E}(\mathrm{~d} y) D_{E}(y) f(y)$. Finally, for any $f \in \overline{\mathscr{C}}_{0}(Y)$ we have

$$
\begin{aligned}
\int \mu_{E}(\mathrm{~d} y) f(y) & =\hat{\mu}_{E}(f) \\
& =\operatorname{tr}[E(f)] \\
& =\int \mu_{E}(\mathrm{~d} y) \operatorname{tr}\left[D_{E}(y)\right] f(y),
\end{aligned}
$$

which implies $\operatorname{tr}\left[D_{E}(y)\right]=1 \mu_{E}$-almost everywhere.

\section{B. Extreme OVEs}

We show here that every extreme POVM in dimension $d$ is concentrated on a finite set of $k \leq d^{2}$ points. This is done by characterizing the set of extreme regular OVEs.

Lemma 2 Let $E \in \mathscr{R}(Y, d)$ be a regular $O V E$, and let $\mu_{E}$ be the finite measure associated to $E$ as in Lemma 1 . If $E$ is extreme, then the associated measure $\mu_{E}$ is concentrated on a finite set of $k \leq d^{2}$ points.

Proof. Let $\mu_{E}$ and $D_{E}$ be the finite measure and the density function associated to $E$ as in Lemma 1 respectively. The density $D_{E} \in L_{\infty}\left(Y, \mu_{E}\right) \otimes \mathrm{M}_{d}$ induces a linear operator $\hat{D}_{E}: \mathrm{M}_{d}^{*} \rightarrow L_{\infty}\left(Y, \mu_{E}\right)$ according to $\hat{D}_{E}(\rho)=(\mathrm{id} \otimes \rho) D_{E}$, id denoting the identity map on $L_{\infty}\left(Y, \mu_{E}\right)$. The dimension of the image of $\hat{D}_{E}$ is clearly bounded by $d^{2}$, which is the dimension of its domain. By absurdum, suppose that $E$ is extreme and the support of the measure $\mu_{E}$ contains more than $d^{2}$ points. Since the space $Y$ is Hausdorff, this implies that the dimension of $L_{\infty}\left(Y, \mu_{E}\right)$ is strictly larger than $d^{2}{ }^{1}$. Hence, there is at least one function $h \in L_{\infty}\left(Y, \mu_{E}\right)$ that is linearly independent from all elements in the image of $\hat{D}_{E}$. The function $h$ can be chosen to be real without loss of generality. Moreover, since $\mu_{E}$ is a finite measure on $Y$, the inclusion $L_{\infty}\left(Y, \mu_{E}\right) \subseteq L_{2}\left(Y, \mu_{E}\right)$ holds, and $S=\left\{\alpha h+\beta \hat{D}_{E}(\rho) \mid \alpha, \beta \in \mathbb{C}, \rho \in \mathrm{M}_{d}^{*}\right\}$ is a $\left(d^{2}+1\right)$-dimensional closed subspace of $L_{2}\left(Y, \mu_{E}\right) \cap L_{\infty}\left(Y, \mu_{E}\right)$. It is then possible to choose a non-zero real function $g \in S$ with $\|g\|_{\infty}<\infty$ that is orthogonal to all elements in the image of $\hat{D}_{E}$, namely

$$
\left\langle g, \hat{D}_{E}(\rho)\right\rangle=\int_{Y} \mu_{E}(\mathrm{~d} y) g(y) \hat{D}_{E}(\rho)(y)=0 .
$$

This implies the decomposition $E=\frac{1}{2}\left(E_{+}+E_{-}\right)$where

$$
E_{ \pm}(f)=E((1 \pm \tau g) f), \quad \tau=\frac{1}{2\|g\|_{\infty}}
$$

We claim that the above decomposition is a nontrivial convex decomposition of $E$, in contradiction with the fact that $E$ is extreme. First, $E_{ \pm}$is a positive map: $E_{ \pm}(f)=E((1 \pm \tau g) f) \geq 0$ for any positive function $f \geq 0$. The normalization $E_{ \pm}\left(\mathbb{1}_{Y}\right)=\mathbb{1}_{d}$ follows from the relation $\rho\left(E_{ \pm}\left(\mathbb{1}_{Y}\right)\right)=\rho\left(E\left(\mathbb{1}_{Y}\right)\right) \pm \tau\left\langle g, \hat{D}_{E}(\rho)\right\rangle=\rho\left(\mathbb{1}_{d}\right)$ holding for any $\rho \in M_{d}^{*}$ due to Eq. (20). Hence, $E_{ \pm}$is an OVE. Finally, the decomposition is nontrivial, namely $E_{+} \neq E_{-}$. Indeed, one has $E_{+}(f)-E_{-}(f)=2 \tau E(f g)$, which cannot be zero for any $f \in \overline{\mathscr{C}}_{0}(Y)$, otherwise using Lemma 1 one would have also

$$
\begin{aligned}
0=\operatorname{tr}[E(f g)] & =\int_{Y} \mu_{E}(\mathrm{~d} y) \operatorname{tr}\left[D_{E}(y)\right] f(y) g(y) \\
& =\int_{Y} \mu_{E}(\mathrm{~d} y) f(y) g(y)=\langle g, f\rangle
\end{aligned}
$$

\footnotetext{
${ }^{1}$ Indeed, for any finite collection of points $\left\{y_{i} \in \operatorname{supp}\left(\mu_{E}\right) \mid i=1, \ldots, k<\infty\right\}$ there is a collection of open neighborhoods $\left\{U_{i} \mid i=1, \ldots, k\right\}$ with $U_{i} \cap U_{j}=\emptyset$ for $i \neq j$. If the support contains more than $d^{2}$ points, then the dimension of $L_{\infty}\left(Y, \mu_{E}\right)$ is clearly larger than $d^{2}$, as the indicator functions of the sets $U_{i}$ are linearly independent elements of $L_{\infty}\left(Y, \mu_{E}\right)$.
} 
for any $f \in \overline{\mathscr{C}_{0}}(Y)$, in contradiction with the fact that $g \in L_{2}\left(Y, \mu_{E}\right)$ is nonzero by construction.

As a consequence of the previous Lemma one can reduce the characterization of extreme OVEs with locally compact Hausdorff space $Y$ to the characterization of extreme OVEs with finite outcome space:

Theorem 2 (Characterization of extreme regular OVEs) Let $Y$ be a locally compact Hausdorff space, and $X$ be a finite set with cardinality $|X|=\min \left\{d^{2},|Y|\right\}$. A regular $O V E E \in \mathscr{R}(Y, d)$ is extreme if and only if there exists an extreme $O V E P \in \mathscr{E}(X, d)$ and an injective function $\varphi \in \mathscr{C}(X, Y)$ such that the following identity holds:

$$
E(f)=P(f \circ \varphi) \quad \forall f \in \overline{\mathscr{C}_{0}}(Y) .
$$

Proof. Suppose that $E$ is extreme. Then, according to Lemma 2 the measure $\mu_{E}$ is concentrated on a finite set of points $\left\{y_{i} \mid i=1, \ldots, k\right\}$ with $k \leq d^{2}$, namely $\mu_{E}(\Delta)=\sum_{i=1}^{k} \chi_{\Delta}\left(y_{i}\right) p_{i}$, with $p_{i} \geq 0, \sum_{i} p_{1}=1$. Using Lemma 1 one obtains

$$
\begin{aligned}
E(f) & =\int_{Y} \mu_{E}(\mathrm{~d} y) D_{E}(y) f(y) \\
& =\sum_{i=1}^{k} p_{i} D_{E}\left(y_{i}\right) f\left(y_{i}\right) \\
& =\sum_{i=1}^{|X|} P_{i} f\left(y_{i}\right) \\
& =P(f \circ \varphi)
\end{aligned}
$$

where $X=\left\{1,2, \ldots, \min \left\{d^{2},|Y|\right\}, P(h)=\sum_{i} h(i) P_{i}\right.$ for any $h \in \mathscr{C}(X)$,

$$
P_{i}= \begin{cases}p_{i} D_{E}\left(y_{i}\right) & i=1, \ldots, k \\ 0 & i=k+1, \ldots,|X|\end{cases}
$$

and $\varphi \in \mathscr{C}(X, Y)$ is any injective function such that $\varphi(i)=y_{i}, \forall i=1, \ldots, k$. Obviously $P$ must be extreme in $\mathscr{E}(X, d)$, otherwise one would obtain a non-trivial convex decomposition of $E$. Conversely, suppose $E$ is as in Eq. (24). Then the measure $\mu_{E}$ associated to $E$ has finite $\operatorname{support} \operatorname{supp}\left(\mu_{E}\right) \subseteq \varphi(X)=\left\{y_{i} \mid i=1, \ldots, \min \left\{d^{2},|Y|\right\}\right\}$. Suppose that $E=1 / 2\left(E_{+}+E_{-}\right)$with $E_{ \pm} \in \mathscr{E}(Y, d)$. Since $E_{ \pm}$are positive maps, we have $E_{ \pm} \leq 2 E \leq 2 \hat{\mu}_{E} \mathbb{1}_{d}$, where $\hat{\mu}_{E}$ is the functional associated to $\mu_{E}$. Due to the Radon-Nikodym theorem for OVEs [Lemma 11] of the Appendix], $E_{ \pm}$admits a density with respect to $\mu_{E}$, whence

$$
\begin{aligned}
E_{ \pm}(f) & =\int_{Y} \mu_{E}(\mathrm{~d} y) D_{ \pm}(y) f(y) \\
& =\sum_{i \in X} p_{i} D_{ \pm}\left(y_{i}\right) f\left(y_{i}\right) \\
& =P_{ \pm}(f \circ \varphi) .
\end{aligned}
$$

upon defining the OVE $P_{ \pm} \in \mathscr{E}(X, d)$ by $P(h)=\sum_{i \in X} p_{i} D_{ \pm}\left(y_{i}\right) h(i), \forall h \in \mathscr{C}(X)$. Moreover, since $Y$ is a locally compact Hausdorff space and $\varphi$ is injective, the mapping $f \mapsto f \circ \varphi$ is surjective on $\mathscr{C}(X)^{2}$. Therefore we have $P(h)=1 / 2\left(P_{+}(h)+P_{-}(h)\right)$ for any $h \in \mathscr{C}(X)$, i.e. $P=1 / 2\left(P_{+}+P_{-}\right)$and, due to extremality of $P, P_{+}=P_{-}=P$. In conclusion, we obtained $E_{+}=E_{-}=E$, i.e. $E$ is extreme.

For any continuous function $\varphi: X \rightarrow Y$, we now define the continuous map $\hat{\varphi}: \mathscr{E}(X, d) \rightarrow \mathscr{E}(Y, d)$, which maps $P \in \mathscr{E}(X, d)$ to the OVE $\hat{\varphi}(P) \in \mathscr{E}(Y, d)$ defined by the relation

$$
\hat{\varphi}(P)(f)=P(f \circ \varphi) \quad \forall f \in \overline{\mathscr{C}_{0}}(Y) .
$$

\footnotetext{
2 Since any locally compact Hausdorff space is completely Hausdorff, for any $i \in X$ there exists a function $f_{i} \in \overline{\mathscr{C}_{0}}(Y)$ that separates $y_{i}$ from the finite set $\left\{y_{j} \mid j \in X, j \neq i\right\}$, namely $f_{i}\left(y_{j}\right)=\delta_{i j}$. As a consequence, $h_{i}(j):=f_{i} \circ \varphi(j)=f_{i}\left(y_{j}\right)=\delta_{i j}$. Since the functions $h_{i}$ are a basis for the finite dimensional vector space $\mathscr{C}(X)$, the map $f \mapsto f \circ \varphi$ is surjective.
} 
We denote by $\mathscr{I}(X, Y)$ the set of injective functions in $\mathscr{C}(X, Y)$, and define a map $\iota_{X, Y}$ that transforms subsets of $\mathscr{E}(X, d)$ into subsets of $\mathscr{E}(Y, d)$ as follows

$$
\iota_{X, Y}(C):=\{\hat{\varphi}(P) \mid \varphi \in \mathscr{I}(X, Y), P \in C\} \quad \forall C \subseteq \mathscr{E}(X, d) .
$$

With this definition, we can state the following

Corollary $\mathbf{2}$ Let $X, Y$ be as in Theorem $\mathbb{2}$, and let $\bar{Y}$ be the one point compactification of $Y$. Then the following equalities hold:

$$
\begin{aligned}
\partial \mathscr{R}(Y, d) & =\iota_{X, Y}(\partial \mathscr{E}(X, d)) \\
\partial \mathscr{E}(Y, d) & =\iota_{X, \bar{Y}}(\partial \mathscr{E}(X, d)) .
\end{aligned}
$$

Moreover, $\partial \mathscr{R}(Y, d)=\partial \mathscr{E}(Y, d) \cap \mathscr{R}(Y, d)$.

Proof. Eq. (33) directly follows from Theorem 2, Eq. (34) follows from Theorem 2 and from the identification $\mathscr{E}(Y, d) \simeq \mathscr{E}(\bar{Y}, d) \equiv \mathscr{R}(\bar{Y}, d)$. Finally, combining Eqs. 333) and wave he the inclusion

$$
\begin{aligned}
\partial \mathscr{R}(Y, d) & =\iota_{X, Y}(\partial \mathscr{E}(X, d)) \subseteq \iota_{X, \bar{Y}}(\partial \mathscr{E}(X, d)) \cap \mathscr{R}(Y, d) \\
& =\partial \mathscr{E}(Y, d) \cap \mathscr{R}(Y, d) .
\end{aligned}
$$

Conversely, an OVE $E \in \partial \mathscr{E}(Y, d)$, given by $E(f)=P(f \circ \varphi)=\sum_{i} f(\varphi(i)) P_{i}$, is regular only if $\varphi(i) \in Y$ for any $i$ such that $P_{i} \neq 0$. Therefore, there exists an injective function $\tilde{\varphi} \in \mathscr{I}(X, Y)$ such that $E(f)=P(f \circ \tilde{\varphi})$, namely $E \in \partial \mathscr{R}(Y, d)$. In conclusion, we have $\partial \mathscr{R}(Y, d)=\partial \mathscr{E}(Y, d) \cap \mathscr{R}(Y, d)$.

The characterization of extreme POVMs immediately follows as a corollary from the previous Theorem:

Corollary 3 (Extreme POVMs) Let $X$ and $Y$ be as in Theorem Q A POVM $M \in \mathscr{M}(Y, d)$ is extreme if and only if there exist an injective function $\varphi \in \mathscr{C}(X, Y)$, and an extreme finite-outcome POVM $P \in \mathscr{M}(X, d)$ such that for any Borel set $\Delta \in \sigma(Y)$

$$
M(\Delta)=\sum_{i \in X} \chi_{\Delta}(\varphi(i)) P_{i}
$$

$\chi_{\Delta}$ denoting the indicator function of $\Delta$.

Remark 3 The above characterization implies that any extreme quantum measurement $M \in \mathscr{M}(Y, d)$ with locally compact outcome space $Y$ can be realized by first performing finite-outcome measurement $\left\{P_{i} \mid i \in X\right\}$, and then, conditionally to outcome $i \in X$, by declaring outcome $\varphi(i) \in Y$. In such a scheme the function $\varphi \in \mathscr{C}(X, Y) \operatorname{simply}$ represents a classical post-processing of the measured data. It is worth stressing that for extreme POVMs such a post-processing must be injective: $\varphi(i)=\varphi(j)$ only if $i=j$.

For the sake of completeness we conclude this Section with a characterization of extreme OVEs in $\mathscr{E}(X, d)$, which coincides with the characterization of extreme finite-outcome POVMs of Ref. (32).

Theorem 3 (Extreme finite-outcome OVEs) Let $P \in \mathscr{E}(X, d)$ be an OVE with finite outcome space, given by $P(h)=\sum_{i} h_{i} P_{i}, P_{i} \in M_{d}$. Denote by $\mathcal{H}_{i}$ the range of $P_{i}$ and by $\mathscr{B}\left(\mathcal{H}_{i}\right)$ the algebra of linear operators on $\mathcal{H}_{i}$. Then, $P$ is extreme if and only if the map $T_{P}: \bigoplus_{i \in X} \mathscr{B}\left(\mathcal{H}_{i}\right) \rightarrow \mathrm{M}_{d}$ given by

$$
T_{P}\left(\bigoplus_{i} A_{i}\right)=\sum_{i \in X} \sqrt{P_{i}} A_{i} \sqrt{P_{i}}
$$

is injective.

Proof. Suppose $P=1 / 2\left(P_{+}+P_{-}\right)$for some $P_{ \pm} \in \mathscr{E}(X, d)$. This implies that $2 P-P_{ \pm} \geq 0$, i.e. $P_{ \pm}$is dominated by $2 P$. Let $\left(\mathcal{H}_{P}, \pi_{P}, V_{P}\right)$ be the minimal Stinespring representation $(31)$ of $P$, given by $\mathcal{H}_{P}=\bigoplus_{i} \mathcal{H}_{i}, \pi_{P}(h)=\bigoplus_{i} h_{i} \mathbb{1}_{\mathcal{H}_{i}}$, and $V_{P}=\sum_{i} \sqrt{P_{i}} \otimes|i\rangle$ (here the tensor with $|i\rangle$ denotes the embedding of $\mathcal{H}_{i}$ in $\mathcal{H}_{P}$ and the operator $\sqrt{P_{i}} \otimes|i\rangle$ is defined by $\left(\sqrt{P_{i}} \otimes|i\rangle\right) \varphi=\left(\sqrt{P_{i}} \varphi\right) \otimes|i\rangle$, for any $\left.\varphi \in \mathbb{C}^{d}\right)$. The Radon-Nikodym theorem for completely positive maps $\left(2 ; 3\right.$; 34) then implies $P_{ \pm}(h)=V_{P}^{\dagger} D_{ \pm} \pi_{P}(h) V_{P}$, for some positive operator $D_{ \pm}$in the commutant of $\pi_{P}$, i.e. in $\bigoplus_{i} \mathscr{B}\left(\mathcal{H}_{i}\right)$. Accordingly, we have $P_{ \pm}(h)=\sum_{i} h_{i} \sqrt{P_{i}} D_{i}^{ \pm} \sqrt{P_{i}}$ with $D_{i}^{ \pm} \in \mathscr{B}\left(\mathcal{H}_{i}\right)$. Since we have $P_{ \pm}\left(\mathbb{1}_{X}\right)=T_{P}\left(D_{ \pm}\right)$, the normalization condition $P_{ \pm}\left(\mathbb{1}_{X}\right)=\mathbb{1}_{d}$ is satisfied with $P_{+} \neq P_{-}$if and only if the map $T_{P}$ is not injective, i.e. $P$ is not extreme if and only if $T_{P}$ is not injective. 


\section{TOPOLOGICAL PROPERTIES OF $\mathscr{E}(Y, d)$ AND $\mathscr{R}(Y, d)$}

Operator valued expectations are elements of the Banach space $\mathscr{B}(Y, d)$ of bounded maps from $\overline{\mathscr{C}_{0}}(Y)$ to $\mathrm{M}_{d}$, which is naturally isomorphic to the Banach space $\overline{\mathscr{C}_{0}}(Y)^{*} \otimes \mathrm{M}_{d}$ :

Lemma 3 Let $V$ denote the Banach space $V=\overline{\mathscr{C}}_{0}(Y) \otimes \mathrm{M}_{d}^{*}$, equipped with the cross norm

$$
\|B\|=\inf \left\{\sum_{i}\left\|f_{i}\right\|\left\|\rho_{i}\right\|_{1} \mid B=\sum_{i} f_{i} \otimes \rho_{i}\right\},
$$

$\|\cdot\|_{1}$ being the norm on $\mathrm{M}_{d}^{*}$. Then, the Banach space $\mathscr{B}(Y, d)$ is isomorphic to the dual Banach space $V^{*}$.

Proof. Any map $E \in \mathscr{B}(Y, d)$ induces a linear functional $\hat{E} \in V^{*}$, which is defined on product vectors by $\hat{E}(f \otimes \rho):=\rho(E(f))$ and uniquely extended on $V$ by linearity. The correspondence $E \mapsto \hat{E}$ is invertible and preserves the norm, i.e. $\|E\|=\|\hat{E}\|_{V^{*}}$ where $\|\hat{E}\|_{V^{*}}=\sup _{B,\|B\|=1}|\hat{E}(B)|$. Indeed, on the one hand we have $\|E\|=\sup _{\rho,\|\rho\|_{1}=1} \sup _{f,\|f\|=1}|\rho(E(f))| \leq \sup _{B,\|B\|=1}|\hat{E}(B)|=\|\hat{E}\|_{V^{*}}$. On the other hand, for any possible decomposition of $B \in V$ as $B=\sum_{i} f_{i} \otimes \rho_{i}$ we have $|\hat{E}(B)|=\left|\sum_{i} \rho_{i}\left(E\left(f_{i}\right)\right)\right| \leq\|E\| \sum_{i}\left\|\rho_{i}\right\|_{1}\left\|f_{i}\right\|$. Taking the infimum over all decompositions we get $\|\hat{E}\|_{V^{*}} \leq\|E\|$, and, therefore, $\|E\|=\|\hat{E}\|_{V^{*}}$.

Owing to the above isomorphisms, in the following we identify the map $E$ with the functional $\hat{E}$ and the set $\mathscr{B}(Y, d)$ with $V^{*}$.

Lemma 4 The convex set $\mathscr{E}(Y, d) \subset V^{*}$ is closed and compact in the weak*-topology.

Proof. Suppose that a net $\left(E_{a}\right)_{a \in A} \subset \mathscr{E}(Y, d)$ converges to the linear functional $E \in V^{*}$ in the weak*-topology, i.e. $\lim _{a} E_{a}(B)=T(B)$ for any $B \in V$. In particular, for $B=f \otimes \rho$ we have $\rho(E(f))=\lim _{a} \rho\left(E_{a}(f)\right)$. Since for any positive function $f \geq 0$ one has $E_{a}(f) \geq 0$ for any $a \in A$, one necessarily has also $E(f) \geq 0$. Similarly, $E_{a}\left(\mathbb{1}_{Y}\right)=\mathbb{1}_{d}, \forall a \in A$ implies $E\left(\mathbb{1}_{Y}\right)=\mathbb{1}_{d}$. This proves that $E$ is an element of $\mathscr{E}(Y, d)$, whence $\mathscr{E}(Y, d)$ is weak ${ }_{-}$ closed. Finally, since $\mathscr{E}(Y, d)$ is contained in the unit ball of $V^{*}$ (see Eq. (9)), it is weak*-compact due to the Banach-Alaoglu theorem.

Lemma 5 If $Y$ is second countable, then the set $\mathscr{E}(Y, d)$ is metrizable.

Proof. Since $Y$ is second countable, also its one point compactification $\bar{Y}$ is second countable. Being a second countable compact space, $\bar{Y}$ is then metrizable due to Urysohn's metrization theorem (36). This implies that the Banach space of continuous functions $\mathscr{C}(\bar{Y})$ is separable $(29)$. Moreover, since the dimension $d$ is finite, the Banach space $V=\mathscr{C}(\bar{Y}) \otimes \mathrm{M}_{d}^{*}$ is also separable. We now invoke the well known result that the unit ball in the dual of a separable Banach space is weak*-metrizable (15). Since $\mathscr{E}(Y, d)$ is a subset of the unit ball in $V^{*}$, it is metrizable.

We conclude with the following useful Lemma about the set of regular OVEs

Lemma 6 The set $\mathscr{R}(Y, d)$ is a $G_{\delta}$-set, namely there exists a sequence of open sets $\left\{U_{n}\right\}$ such that $\mathscr{R}(Y, d)=\bigcap_{n} U_{n}$. Moreover, if a regular $O V E E \in \mathscr{R}(Y, d)$ is the barycenter of $\mathscr{E}(Y, d)$ with respect to a probability measure $p_{E}$, then $\mathscr{R}(Y, d)$ has unit measure, i.e. $p_{E}(\mathscr{R}(Y, d))=1$.

Proof. Definition 4 of a regular OVE is equivalent to the condition

$$
\sup \left\{\tau(E(f)) \mid f \in \mathscr{C}_{0}(Y), 0 \leq f \leq \mathbb{1}_{Y}\right\}=1,
$$

where $\tau=\operatorname{tr} / d$ is the normalized trace on $\mathrm{M}_{d}$. Denote by $\mathscr{S}_{n} \subseteq \mathscr{E}(Y, d)$ the set of OVEs $E \in \mathscr{E}(Y, d)$ such that

$$
\sup \left\{\tau(E(f)) \mid f \in \mathscr{C}_{0}(Y), 0 \leq f \leq \mathbb{1}_{Y}\right\} \leq 1-\frac{1}{n}
$$

The set $\mathscr{I}_{n}$ is a weak* ${ }^{*}$-closed subset of $\mathscr{E}(Y, d)$. If an OVE $E \in \mathscr{E}(Y, d)$ is not regular, then it must be in one of the sets $\mathscr{S}_{n}$ for some $n \in \mathbb{N}$, namely

$$
\mathscr{E}(Y, d) \backslash \mathscr{R}(Y, d)=\bigcup_{n} \mathscr{S}_{n}
$$


Since $\mathscr{R}(Y, d)=\bigcap_{n}\left(\mathscr{E}(Y, d) \backslash \mathscr{S}_{n}\right)$ and the each set $U_{n}:=\mathscr{E}(Y, d) \backslash \mathscr{S}_{n}$ is open, $\mathscr{R}(Y, d)$ is a $G_{\delta}$-set. In particular, $\mathscr{R}(Y, d)$ is measurable. Moreover, for any $f \in \mathscr{C}_{0}(Y), 0 \leq f \leq \mathbb{1}_{Y}$ we have the following bound

$$
\begin{aligned}
\tau(E(f)) & =\int_{\mathscr{E}(Y, d)} p_{E}(\mathrm{~d} F) \tau(F(f)) \\
& =\int_{\mathscr{S}_{n}} p_{E}(\mathrm{~d} F) \tau(F(f))+\int_{\mathscr{E}(Y, d) \backslash \mathscr{S}_{n}} p_{E}(\mathrm{~d} F) \tau(F(f)) \\
& \leq(1-1 / n) p_{E}\left(\mathscr{S}_{n}\right)+\left(1-p_{E}\left(\mathscr{S}_{n}\right)\right) \\
& =1-p_{E}\left(\mathscr{S}_{n}\right) / n
\end{aligned}
$$

Taking the supremum with respect to $f$ and using the regularity condition (39), we then obtain $p_{E}\left(\mathscr{S}_{n}\right)=0$ for any $n$. As a consequence, $\mathscr{R}(Y, d)$ has unit measure.

\section{BARYCENTRIC DECOMPOSITION}

\section{A. Case of second countable outcome spaces}

According to Lemmas 4 and 5 , the set $\mathscr{E}(Y, d)$ is compact metrizable set. Choquet's theorem (5; 8) then implies the following:

Lemma 7 Let $Y$ be a second countable locally compact Hausdorff space. Any OVE E $\in \mathscr{E}(Y, d)$ is the barycenter of $\partial \mathscr{E}(Y, d)$ with respect to a suitable probability measure $p_{E}$.

Proof. Direct application of Choquet's theorem.

We now combine the Choquet representation with the regularity condition:

Theorem 4 (Barycentric representation of regular OVEs) Let $Y$ be a locally compact second countable Hausdorff space. Then, any regular $O V E E \in \mathscr{R}(Y, d)$ is the barycenter of the set $\partial \mathscr{R}(Y, d)$ with respect to a probability distribution $p_{E}$.

Proof. By Lemma 7 any OVE $E \in \mathscr{E}(Y, d)$ is the barycenter of the set $\partial \mathscr{E}(Y, d)$ with respect to a probability measure $p_{E}$. On the other hand, since $E$ is regular, Lemma 6 requires the set $\mathscr{R}(Y, d)$ to have unit measure. Finally, by Corollary 2 we have $\partial \mathscr{R}(Y, d)=\partial \mathscr{E}(Y, d) \cap \mathscr{R}(Y, d)$. Since both $\partial \mathscr{E}(Y, d)$ and $\mathscr{R}(Y, d)$ are measurable sets with unit measure, also their intersection enjoys this property.

Owing to the affine bijection established by Theorem 1 the present result can be readily translated into a Choquet representation of POVMs in finite dimensional Hilbert spaces.

Corollary 4 (Barycentric representation of POVMs) Let $Y$ be a locally compact second countable Hausdorff space. Then, any POVM $M \in \mathscr{M}(Y, d)$ is the barycenter of the set $\partial \mathscr{M}(Y, d)$ with respect to a probability distribution $p_{M}$, namely

$$
M(\Delta)=\int_{\partial \mathscr{M}(Y, d)} p_{M}(\mathrm{~d} P) P(\Delta) \quad \forall \Delta \in \sigma(Y)
$$

Remark 4 The above Choquet representation, once combined with the characterization of extreme POVMs of Corollary 3. shows that quantum measurements with second-countable outcome space can always be interpreted as randomizations of extreme finite-outcome measurements, corresponding to operator valued measures concentrated on $k \leq d^{2}$ points. It is worth stressing that essentially all outcome spaces that are relevant for applications in Quantum Mechanics are separable and metrizable, and that for locally compact Hausdorff spaces these two conditions are equivalent to second countability, due to Urysohn's metrization theorem. 


\section{B. General case}

If the outcome space $Y$ is not second countable, the set $\mathscr{E}(Y, d)$ is generally not metrizable. In this situation, Choquet's theorem cannot be applied, and a barycentric decomposition only in terms extreme points might not be possible. However, since the set $\mathscr{E}(Y, d)$ is compact in the weak*-topology (Lemma 4), we can still exploit KreinMilman theorem, thus getting the following

Lemma 8 Let $Y$ be a locally compact Hausdorff space, and $\overline{\partial \mathscr{E}(Y, d)}$ be the weak*-closure of $\partial \mathscr{E}(Y, d)$. Any OVE $E \in \mathscr{E}(Y, d)$ is the barycenter of the set $\overline{\partial \mathscr{E}(Y, d)}$ with respect to a probability measure $p_{E}$.

Proof. Direct consequence of Krein-Milman theorem [Lemma 12 of the Appendix].

Remark 5 Notice that in most situations the set $\partial \mathscr{E}(Y, d)$ is not weak*-closed. For example, take $d=2$ and $Y \equiv X=\{1,2,3,4\}$, and consider the OVEs $E_{a}$ defined by $E_{a}(h)=\sum_{i} h_{i} E_{i, a}, \forall h \in \mathscr{C}(X)$ with

$$
\begin{aligned}
& E_{1, a}=\left(\mathbb{1}+\cos a \sigma_{x}+\sin a \sigma_{y}\right) / 4 \\
& E_{2, a}=\left(\mathbb{1}+\cos a \sigma_{x}-\sin a \sigma_{y}\right) / 4 \\
& E_{3, a}=\left(\mathbb{1}-\cos a \sigma_{x}+\sin a \sigma_{z}\right) / 4 \\
& E_{4, a}=\left(\mathbb{1}-\cos a \sigma_{x}-\sin a \sigma_{z}\right) / 4,
\end{aligned}
$$

where $\sigma_{x}=\left(\begin{array}{ll}0 & 1 \\ 1 & 0\end{array}\right), \sigma_{y}=\left(\begin{array}{rr}0 & -i \\ i & 0\end{array}\right), \sigma_{z}=\left(\begin{array}{rr}1 & 0 \\ 0 & -1\end{array}\right)$. Using Theorem 3 it is immediate to verify that the OVE $E_{a}$ is extreme for any $a \in(0, \pi / 4]$, while the limit $E=\lim _{a \rightarrow 0} E_{a}$ is not extreme, namely $\partial \mathscr{E}(Y, d)$ is not closed, whence the decomposition of Lemma 8 necessarily involves some non-extreme OVEs.

Theorem 5 (Barycentric decomposition of regular OVEs) Let $Y$ be a locally compact Hausdorff space, and $\mathscr{F}(Y, d)$ be the intersection

$$
\mathscr{F}(Y, d)=\overline{\partial \mathscr{E}(Y, d)} \cap \mathscr{R}(Y, d)
$$

Then, any regular $O V E E \in \mathscr{R}(Y, d)$ is the barycenter of the set $\mathscr{F}(Y, d)$ with respect to a suitable probability measure $p_{E}$.

Proof. By Lemma 8, any OVE $E$ is the barycenter of the set $\overline{\partial \mathscr{E}(Y, d)}$ with respect to a probability measure $p_{E}$. Combining this fact with Lemma 6 we immediately obtain the thesis.

Although the set $\mathscr{F}(Y, d)$ contains also OVEs that are not extreme, it is simple to realize that it only contains OVEs that correspond to POVMs concentrated on a finite set of points of $Y$. We now conclude the paper by proving this fact, by first showing that all OVEs in $\overline{\partial \mathscr{E}(Y, d)}$ correspond to POVMs concentrated on a finite set of points of $\bar{Y}$, and then using the regularity condition. Let us identify $\mathscr{C}(X, Y)$ with $X \times Y \subseteq X \times \bar{Y}$ and equip it with the product topology. Accordingly, $\overline{\mathscr{I}(X, Y)}$ denotes the closure of the set of injective functions in $\mathscr{C}(X, Y)$. Define the map $\bar{\iota}_{X, Y}$ transforming subsets of $\mathscr{E}(X, d)$ into subsets of $\mathscr{E}(X, d)$ as follows

$$
\bar{\iota}_{X, Y}(C):=\{\hat{\varphi}(P) \mid \varphi \in \overline{\mathscr{I}(X, Y)}, P \in C\},
$$

where the map $\hat{\varphi}$ is defined as in Eq. (31). We then have the following:

Lemma 9 Let $X$ and $Y$ be as in Theorem [2, and $\iota_{X, Y}$ and $\bar{\iota}_{X, Y}$ be the maps defined in Eqs. (32) and (49), respectively. Then, for any subset $C \subseteq \mathscr{E}(X, d)$, one has

$$
\overline{\iota_{X, Y}(C)}=\bar{\iota}_{X, Y}(\bar{C})
$$

Proof. Let $E$ be a point of $\overline{\iota_{X, Y}(C)}$, and take a net $\left(E_{a}\right)_{a \in A} \subset \iota_{X, Y}(C)$ converging to $E$. Since $E_{a} \in \iota_{X, Y}(C)$, one has $E_{a}(f)=P_{a}\left(f \circ \varphi_{a}\right)$, with $P_{a} \in C$ and $\varphi_{a} \in \mathscr{I}(X, Y)$. Moreover, since $\bar{C}$ is compact, the net $\left(P_{a}\right)_{a \in A} \subset \bar{C}$ will have a cluster point $P \in \bar{C}$. Similarly, the net $\left(\varphi_{a}\right)_{a \in A} \subset \overline{\mathscr{I}(X, Y)}$ will have a cluster point $\varphi \in \overline{\mathscr{I}(X, Y)}$. We can then choose a subnet $\left(E_{b}\right)_{b \in B}$ such that $\lim _{b} P_{b}=P$ and $\lim _{b} \varphi_{b}=\varphi$, thus obtaining

$$
E(f)=\lim _{b} E_{b}(f)=\lim _{b} P_{b}\left(f \circ \varphi_{b}\right)=P(f \circ \varphi) .
$$


To evaluate the limit we used the fact that $\mathscr{E}(X, d)$ is finite dimensional, whence the weak*-convergence of the net $\left(P_{b}\right)_{b \in B}$ is equivalent to norm convergence. The above equation proves that $E$ is in $\bar{\iota}_{X, Y}(\bar{C})$, namely $\overline{\iota_{X, Y}(C)} \subseteq$ $\bar{\iota}_{X, Y}(\bar{C})$. Conversely, let $E$ be a point in $\bar{\iota}_{X, Y}(\bar{C})$, defined by $E(f)=P(f \circ \varphi)$, with $P \in \bar{C}$ and $\varphi \in \overline{\mathscr{I}(X, Y)}$. Take a net $\left(P_{a}\right)_{a \in A} \subseteq C$ such that $\lim _{a} P_{a}=P$ and a net of injective functions $\left(\varphi_{b}\right)_{b \in B} \subseteq X \times Y$ such that $\lim _{b} \varphi_{b}=\varphi$. Let us equip $A \times B$ with the product order, and define the net $E_{a, b} \in \iota_{X, Y}(C)$ by $E_{a, b}(f):=P_{a}\left(f \circ \varphi_{b}\right)$. Clearly, the net $\left(E_{a, b}\right)_{(a, b) \in A \times B}$ converges to $E$, whence $E \in \overline{\iota_{X, Y}(C)}$. This proves that $\bar{\iota}_{X, Y}(\bar{C}) \subseteq \overline{\iota_{X, Y}(C)}$.

As a consequence, we have the following characterization:

Lemma 10 The closure of the set $\partial \mathscr{E}(Y, d)$ is given by

$$
\overline{\partial \mathscr{E}(Y, d)}=\bar{\iota}_{X, \bar{Y}}(\overline{\partial \mathscr{E}(X, d)}),
$$

namely every $E \in \overline{\partial \mathscr{E}(Y, d)}$ is of the form

$$
E(f)=P(f \circ \varphi) \quad \forall f \in \mathscr{C}_{0}(Y)
$$

for some suitable $O V E P \in \mathscr{E}(X, d)$ and some suitable function $\varphi \in \mathscr{C}(X, \bar{Y})$, obtained as a limit of injective functions.

Proof. By Corollary 2 we have $\partial \mathscr{E}(Y, d)=\iota_{X, \bar{Y}}(\partial \mathscr{E}(X, d))$. Application of Lemma 9 then yields the thesis.

Theorem 6 (Structure of the set $\mathscr{F}(Y, d)$ ) Let

$$
\mathscr{K}(X, Y)=\overline{\mathscr{I}(X, Y)} \cap \mathscr{C}(X, Y)
$$

be the set of continuous functions from $X$ to $Y$ that are limits of injective functions. Then, the set $\mathscr{F}(Y, d)$ defined in Eq. [48) is given by

$$
\begin{aligned}
\mathscr{F}(Y, d)=\{E \in \mathscr{E}(Y, d) \mid & E(f)=P(f \circ \varphi), \\
& \varphi \in \mathscr{K}(X, Y), P \in \overline{\partial \mathscr{E}(X, d)}\}
\end{aligned}
$$

Proof. By definition, $\mathscr{F}(Y, d)=\overline{\partial \mathscr{E}(Y, d)} \cap \mathscr{R}(Y, d)$. On the other hand, by Lemma 10 an OVE $E$ is in $\overline{\partial \mathscr{E}(Y, d)}$ iff if has the form

$$
E(f)=P(f \circ \varphi)=\sum_{i \in X} P_{i} f(\varphi(i))
$$

with $P \in \overline{\partial \mathscr{E}(X, d)}$ and $\varphi \in \overline{\mathscr{I}(X, \bar{Y})}$. Clearly, an OVE $E$ in $\overline{\partial \mathscr{E}(Y, d)}$ is regular iff the function $\varphi$ in Eq. (56)) satisfies $\varphi(X) \subseteq Y$, namely, iff $\varphi \in \overline{\mathscr{I}(X, \bar{Y})} \cap \mathscr{C}(X, Y)$. We now claim that $\overline{\mathscr{I}(X, \bar{Y})} \cap \mathscr{C}(X, Y) \equiv \mathscr{K}(X, Y)$. Indeed, we have the inclusion $\mathscr{K}(X, Y)=\overline{\mathscr{I}(X, Y)} \cap \mathscr{C}(X, Y) \subseteq \overline{\mathscr{I}(X, \bar{Y})} \cap \mathscr{C}(X, Y)$. Viceversa, let $\varphi$ be in $\overline{\mathscr{I}(X, \bar{Y})} \cap \mathscr{C}(X, Y)$ and $\left(\varphi_{a}\right)_{a \in A} \subseteq \mathscr{I}(X, \bar{Y})$ be a net of injective functions such that $\lim _{a} \varphi_{a}=\varphi$. Since the topology of $\mathscr{C}(X, \bar{Y}) \simeq X \times \bar{Y}$ contains the topology of $\mathscr{C}(X, Y)=X \times Y$, for any neighborhood $U \subseteq \mathscr{C}(X, Y)$ of $\varphi$ we have that the net $\left(\varphi_{a}\right)_{a \in A}$ must eventually be in $U$. Hence, $\varphi$ is the limit of a net of injective functions in $\mathscr{I}(X, Y)$ as well. Therefore, we have $\varphi \in \overline{\mathscr{I}(X, Y)} \cap \mathscr{C}(X, Y)=\mathscr{K}(X, Y)$, thus proving the reverse inclusion.

Any OVE in $\mathscr{F}(Y, d)$ corresponds to a POVM concentrated on $|X| \leq d^{2}$ points of $Y$. Indeed, we have

$$
E(f)=P(f \circ \varphi)=\sum_{i=1}^{|X|} f(\varphi(i)) P_{i}=\int_{Y} M(\mathrm{~d} y) f(y),
$$

where $M$ is the POVM defined by $M(\Delta):=\sum_{i=1}^{d^{2}} \chi_{\Delta}\left(y_{i}\right) P_{i}$ for any Borel set $\Delta$. The barycentric decomposition for POVMs is the given by the following: 
Corollary 5 Let $Y$ be a locally compact Hausdorff space, and let $\mathscr{Q}(Y, d)$ be the subset of $\mathscr{M}(Y, d)$ defined by

$$
\begin{array}{r}
\mathscr{Q}(Y, d)=\left\{M \in \mathscr{M}(Y, d) \mid M(\Delta)=\sum_{i=1}^{d^{2}} \chi_{\Delta}(\varphi(i)) P_{i},\right. \\
\varphi \in \mathscr{K}(X, Y), P \in \overline{\partial \mathscr{M}(X, d)}\}
\end{array}
$$

Then, any POVM $M \in \mathscr{M}(Y, d)$ is the barycenter of the set $\mathscr{Q}(Y, d)$ with respect to a probability distribution $p_{M}$, namely,

$$
M(\Delta)=\int_{\mathscr{Q}(Y, d)} p_{M}(\mathrm{~d} P) P(\Delta)
$$

for any Borel set $\Delta$.

The barycentric representation of POVMs with locally compact Hausdorff space allows one to interpret quantum measurements on finite dimensional systems as randomizations of measurements with $k \leq d^{2}$ outcomes, thus providing a rigorous proof of the fact that in finite dimensions continuous spectrum is equivalent to continuous classical randomness controlling the choice of the measuring apparatus.

Acknoledgements. GC gratefully acknowledges financial support from the European Community through the project CORNER.

\section{APPENDIX}

For completeness of the presentation, in the following we provide the proofs of two standard results, the former on the existence of densities for OVEs and the latter on barycentric decompositions in locally convex spaces.

\section{APPENDIX A: Radon-Nikodym theorem for OVEs}

The following Radon-Nikodym theorem for OVEs is equivalent to the existence of a density for POVMs in finite dimensions, which in turn is a consequence of the Radon-Nikodym theorem for quantum instruments (13; 23; 26).

Lemma 11 Let $\mu$ be a finite regular measure on $Y$ and let $T \in \mathscr{E}(Y, d)$ be an $O V E$ satisfying the dominance condition $T \leq \hat{\mu} \mathbb{H}, \hat{\mu} \in \overline{\mathscr{C}}_{0}(Y)^{*}$ being the positive functional associated to $\mu$. Then, there exists a unique positive operator density $D \in L_{\infty}(Y, \mu) \otimes \mathrm{M}_{d}$ such that

$$
T(f)=\int \mu(\mathrm{d} y) f(y) D(y) .
$$

Proof. Since $\hat{\mu}$ is a positive functional, $S=\hat{\mu} \mathbb{1}$ is a completely positive (CP) map. Moreover, due to the dominance condition, $S-T$ is also a CP-map. The Radon-Nikodym Theorem for CP-maps (2; 3; 34) then implies that $T(f)=$ $V_{S}^{*} \pi_{S}(f) D V_{S}$, where $\left(\mathcal{H}_{S}, \pi_{S}, V_{S}\right)$ is the minimal Stinespring representation of $S$, and $D$ is a unique positive operator in the commutant of $\pi_{S}\left(\overline{\mathscr{C}_{0}}(Y)\right)$. The minimal Stinespring representation of $S$ is easily obtained here by the GNS representation of $\hat{\mu}$, given by $\left(\mathcal{H}_{\hat{\mu}}, \pi_{\hat{\mu}}, \Omega_{\hat{\mu}}\right)$. Indeed, the Hilbert space $\mathcal{H}_{S}$ can be identified with $\mathcal{H}_{\hat{\mu}} \otimes \mathbb{C}^{d}$, the representation $\pi_{S}$ with $\pi_{\hat{\mu}} \otimes \mathbb{1}_{d}$, and the isometry $V_{S}$ is defined by

$$
V_{S} \psi=\Omega_{\hat{\mu}} \otimes \psi \quad \forall \psi \in \mathbb{C} .
$$

Therefore, we have

$$
\begin{aligned}
\left\langle\psi_{1}, T(f) \psi_{2}\right\rangle & =\left\langle\psi_{1}, V_{S}^{*} \pi_{S}(f) D V_{S} \psi_{2}\right\rangle \\
& =\left\langle\Omega_{\hat{\mu}} \otimes \psi_{1}, D\left(\pi_{\hat{\mu}}(f) \otimes \mathbb{1}_{d}\right) \Omega_{\hat{\mu}} \otimes \psi_{2}\right\rangle \quad \forall \psi_{1}, \psi_{2} \in \mathbb{C}^{d} .
\end{aligned}
$$


Finally, the GNS Hilbert space $\mathcal{H}_{\hat{\mu}}$ can be identified with $L_{2}(Y, \mu)$, where $\Omega_{\hat{\mu}}$ is the constant function, and $\pi_{\hat{\mu}}$ represents the function $f \in \overline{\mathscr{C}_{0}}(Y)$ by a multiplication operator. With this identification, the commutant of $\pi_{\hat{\mu}}\left(\overline{\mathscr{C}_{0}}(Y)\right) \otimes \mathbb{1}$ is $L_{\infty}(Y, \hat{\mu}) \otimes \mathrm{M}_{d}{ }^{3}$. Therefore, the positive operator $D$ is an operator valued function, yielding

$$
\left\langle\psi_{1}, T(f) \psi_{2}\right\rangle=\int \mu(\mathrm{d} y)\left\langle\psi, D(y) \psi_{2}\right\rangle f(y) \quad \forall \psi_{1}, \psi_{2} \in \mathbb{C}^{d}
$$

which implies the identity $T(f)=\int \mu(\mathrm{d} y) D(y) f(y)$.

\section{APPENDIX B: Barycentric decomposition from Krein-Milman Theorem}

Lemma 12 Let $K$ be a compact subset of a locally convex vector space $X$. Denote with $\overline{\partial K}$ the closure of $\partial K$. Then, any point $x \in K$ is the barycenter of $\overline{\partial K}$ with respect to a suitable probability measure $p_{x}$, namely the relation

$$
f(x)=\int_{\frac{\partial K}{\partial K}} p_{x}(\mathrm{~d} E) f(E)
$$

holds for any function $f \in \mathscr{C}(K)$.

Proof. By Krein-Milman Theorem (35), any $x \in K$ is in the closure of the convex hull of $\partial K$, i.e. that there exists a net $\left(x_{a}\right)_{a}$ contained in the convex hull such that $\lim _{a} x_{a}=x$. Equivalently, $f\left(x_{a}\right)=\sum_{i} p_{i}^{(a)} f\left(x_{i}^{(a)}\right):=\hat{p}^{(a)}(f)$ for any $f \in \mathscr{C}(K)$, where $\left\{p_{i}^{(a)}\right\}$ are probabilities and $\left\{x_{i}^{(a)}\right\}$ is a finite set of points in $\partial K$. Clearly, the restriction of the functional $\hat{p}_{a}$ to the $\mathrm{C}^{*}$-algebra $\mathscr{C}(\overline{\partial K})$ is a state, i.e. a positive normalized functional. Since the set of states is compact, the net $\left(\hat{p}_{a}\right)_{a \in A}$ must have a cluster point $p_{x}$ within it. We then have $f(x)=\lim _{a} f\left(x_{a}\right)=\lim _{a} \hat{p}_{a}(f)=$ $\hat{p}_{x}(f)=\int \frac{\partial_{\partial K}}{p_{x}}(\mathrm{~d} E) f(E), p_{x}$ being the probability distribution on $\overline{\partial K}$ associated to $\hat{p}_{x}$ by Riesz-Markov theorem.

\section{References}

[1] Ali, S. T. and Englis, M.: Quantization methods: a guide for physicists and analysts, Rev. Math. Phys. 17, 391-490 (2005).

[2] Arveson, W. B.: Subalgebras of C*-algebras, Acta Math. 123, 141-224 (1969)

[3] Belavkin, V. P. and Staszewski, P.: A Radon-Nikodym theorem for completely positive maps, Rep. Math. Phys. 24, 49-55 (1986)

[4] Berberian, S. K.: Notes on Spectral Theory, Van Nostrand, Princeton, 1966.

[5] Bishop, E. and De Leeuw, K.: The representation of linear functionals by measures on sets of extreme points, Annales de l'institut Fourier 9, 305-331 (1959).

[6] Busch, P., Lathi, P., and Mittelstaedt, P.: The Quantum Theory of Measurement, Springer, 1991

[7] Bužek, V., Derka, R. and Massar, S.: Optimal quantum clocks. Phys. Rev. Lett. 82, 2207 (1999)

[8] Choquet, G., Existence des représentations intégrales ou moyen des points extrémaux dans les cônes convexes, C. R. Acad. Sci., Paris, 243, 699-702 (1956)

[9] Conway, J. B.: A Course in Functional Analysis, Springer-Verlag, New York, Berlin, Heidelberg, Tokyo, 1985

[10] Chiribella, G., D'Ariano, G. M., Perinotti, P., and Sacchi, M. F.: Efficient use of quantum resources in the transmission of a reference frame. Phys. Rev. Lett. 93, 180503 (2004)

[11] Chiribella, G., D'Ariano, G. M. and Schlingemann, D.-M.: How continuous-outcome measurements in finite dimensions are actually discrete. Phys. Rev. Lett. 98, 190403 (2007)

[12] D'Ariano, G. M., Lo Presti P., and Perinotti. P: Classical randomness in quantum measurements, J. Phys. A: Math. Gen. 385979 (2005)

[13] Davies, E. B.: The Quantum Theory of Open Systems, Academic Press, London, 1976

[14] Davies, E.B., Lewis, J.T.: An operational approach to quantum probability. Comm. Math. Phys. 17, 239-260 (1970).

[15] Dunford, N. and Schwartz, J. T.: Linear Operators, Interscience Publishers, Inc., 1958, p. 424.

[16] Helstrom, C. W.: Quantum Detection and Estimation Theory, Academic Press, New York, 1976

\footnotetext{
${ }^{3}$ Due to the identification $\overline{\mathscr{C}}_{0}(Y) \simeq \mathscr{C}(\bar{Y})$, the commutant of $\pi_{\hat{\mu}}$ coincides with $L_{\infty}(\bar{Y}, \mu)$. On the other hand, since $\hat{\mu}$ is regular one has $L_{\infty}(\bar{Y}, \mu) \equiv L_{\infty}(Y, \mu)$.
} 
[17] Holevo, A. S.: Probabilistic and Statistical Aspects of a Quantum Theory, North Holland, Amsterdam, 1982

[18] Holevo, A.S: An analog of the statistical decision theory in the noncommutative probability theory. Trans. Moscow Mat. Soc. 26 133-149 (1972), AMS Trans. (1974)

[19] Holevo, A. S.: Statistical definition of observable and the structure of statistical models. Rep. Math. Phys. 22 (3), 385-407 (1985)

[20] Holevo, A.S.: Covariant measurements and uncertainty relations. Rep. Math . Phys. 16, 385 (1979)

[21] Holevo, A. S.: Bounds for the quantity of information transmitted by a quantum channel. Probl. Inf. Transm. 9 (3), 177-183 (1973)

[22] Holevo, A. S.: Lectures on Statistical Structure of Quantum Theory (1999).

[23] Holevo, A. S., Radon-Nikodym derivatives of quantum instruments, J. Math. Phys. 39, 1373-1387 (1998)

[24] Yuen, H.P., Shapiro, J.H.: Optical communication with two-photon coherent states-Part III: Quantum measurements realizable with photoemissive detectors. IEEE Trans. Inf. Theory 26, 78 (1980)

[25] Landsman, N. P.: Mathematical Topics between Classical and Quantum Mechanics, Springer Monographs in Mathematics, Springer-Verlag, New York, 1998.

[26] Ozawa, M.: Quantum measuring processes of continuous observables, J. Math. Phys. 25, 79-87 (1984)

[27] Paulsen, V. I.: Completely bounded maps and dilations, Pitman Research Notes in Mathematics Series, 146. Longman Scientific \& Technical, Harlow; John Wiley \& Sons, Inc., New York, 1986

[28] Kelley, P.L., Kleiner, W.H.: Theory of electromagnetic field measurement and photoelectric counting. Phys. Rev. 136A, $316(1964)$

[29] Krein, M., and Krein, S. , On an inner characteristic of the set of all continuous functions defined on a bicompact Hausdorff space, C. R. Acad. Sci. URSS. 27, 427-430 (1940).

[30] Parthasarathy, K. R. : Extremal decision rules in quantum hypothesis testing. Infinite Dimensional Analysis, Quantum Probability and Related Topics, 2, 557 (1999)

[31] Stinespring, W. F.: Positive Functions on C*-Algebras, Proc. Amer. Math. Soc. 6, 211 (1955).

[32] E. Störmer, in Foundations of Quantum Mechanics and Ordered Linear Spaces, A. Hartkämper and H. Neumann eds. Lecture Notes in Physics bf 29, Springer Verlag, Berlin, 1974

[33] Reed, M. and Simon, B.: Methods of modern mathematical physics, Volume I, Academic Press INC., London, 1980

[34] Raginsky, M.: Radon-Nikodym derivatives of quantum operations, J. Math. Phys. 44, 5003-5019.

[35] Royden, H. L.: Real Analysis, Prentice-Hall, Englewood Cliffs, New Jersey, 1988

[36] Urysohn, P.: Zum Metrisationsproblem, Math. Ann. 94, 309-315 (1925)

[37] von Neumann, J.: Mathematical Foundations of Quantum Mechanics, Princeton University Press, Princeton, NJ, 1955.

[38] Wegge-Olsen, N. E.: K-Theory and C-*-Algebras, Oxford University Press, Oxford, 1993. 